\title{
Apollonius' Problem: A Study of Solutions and Their Connections
}

\author{
David Gisch and Jason M. Ribando \\ Department of Mathematics \\ University Northern lowa \\ Cedar Falls, lowa 50614-0506 USA
}

Received:August 15, 2003 Accepted: February 29, 2004

\begin{abstract}
In Tangencies Apollonius of Perga showed how to construct a circle that is tangent to three given circles. More generally, Apollonius' problem asks to construct the circle which is tangent to any three objects that may be any combination of points, lines, and circles. The case when all three objects are circles is the most complicated case since up to eight solution circles are possible depending on the arrangement of the given circles. Within the last two centuries, solutions have been given by J. D. Gergonne in 1816, by Frederick Soddy in 1936, and most recently by David Eppstein in 2001. In this report, we illustrate the solution using the geometry software Cinderella ${ }^{\mathrm{TM}}$, survey some connections among the three solutions, and provide a framework for further study.
\end{abstract}

\section{INTRODUCTION}

Apollonius of Perga was known as 'The Great Geometer'. He should not be confused with other Greek scholars named Apollonius, for it was a common name. Little is known of his life except that he was born in Perga, Pamphylia, which today is known as Murtina, or Murtana, and is now in Antalya, Turkey. The years 262 to 190 B.C. have been suggested for his life [1-3]. It is commonly believed that Apollonius went to Alexandria where he studied under the followers of Euclid and possibly taught there later.

This paper focuses on a problem solved by Apollonius in his book Tangencies. Apollonius' works have had a great influence on the development of mathematics [4]. In particular, his famous book Conics introduced terms which are familiar to us today such as parabola, ellipse and hyperbola. In Book IV of the Elements, Euclid details how to construct a circle tangent to three sides of a given triangle (Proposition 4) and how to construct a circle

containing three noncollinear points (Proposition 5) [5, p. 182]. The latter construction is accomplished by finding the intersection point of the perpendicular bisectors of any two sides of the triangle with the three given points as vertices. In Tangencies Apollonius poses a generalization to Euclid's two propositions: given any three points, lines or circles in the plane, construct a circle which contains the points and is tangent to the lines and circles. Apollonius enumerated the ten combinations of points, lines and circles and solved the cases not already solved by Euclid [6, p.182]. The case when all three objects are circles is the most complicated of the ten cases since up to eight solution circles are possible depending on the arrangement of the circles (see Figure 1).

Since no copy of Apollonius' Tangencies has survived the ages, Pappus of Alexandria deserves credit for eternally linking Apollonius' name with the tangents problem. Pappus, who lived some five centuries after Apollonius and is known 


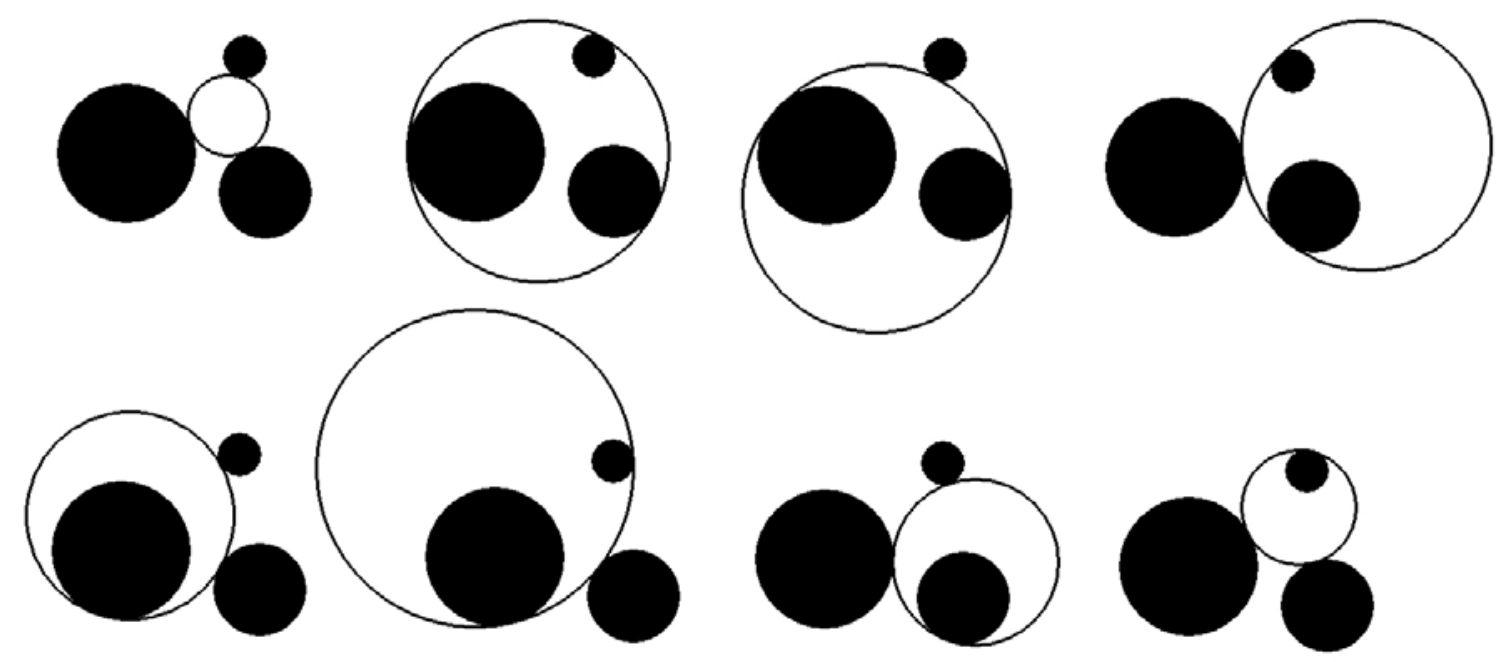

Figure 1. Eight solution circles to Apollonius' problem.

mostly for his encyclopedic recording and commenting of Greek mathematics, wrote a "Treasury of Analysis" in Book VII of his Mathematical Collections dedicated to works of Euclid, Aristaeus, and Apollonius. Here he succinctly states Apollonius' problem, acknowledges the ten cases, and provides a compass and straightedge solution for at least one solution circle [6, p. 182].

Excepting Arabic reconstructions of Apollonius' works, Apollonius' problem lay dormant in the literature until François Viète (1540-1603) restored the Tangencies in 1603. Having unveiled Apollonius' solution, Viète challenged Adrianus Romanus to draw a circle tangent to three given circles but was disappointed by Romanus' use of conics. A century later Newton also went beyond compass and straightedge solutions by employing hyperbolas [7]. Agreeing with Viète's preference for compass and straightedge solutions, we are motivated to include three related constructions under the same cover: the Euler-Gergonne-Soddy triangle, which contains the centers of the two solution circles in the special case when the circles are mutually tangent or "kissing;" a solution found by David Eppstein in 2001 for the same special case; and an adaptation to Gergonne's analytic solution which constructs all eight solution circles in the general case for three circles in generic position. We conclude with some connections among the three solutions and provide a framework for further study beyond the scope of this paper.

\section{THE EULER-GERGONNE-SODDY TRIANGLE OF A TRIANGLE}

A special case of Apollonius' problem is known today as the three coins problem, or kissing coins problem. In this variant, the three circles, of possibly different radii, are taken to be mutually tangent. There are two solutions to this special case of Apollonius' problem: a small circle where all three given circles are externally tangent, and a large circle where the three given circles are internally tangent. In 1643 Renè Descartes sent a letter to Princess Elisabeth of Bohemia in which he provided a solution to this special case of Apollonius' problem. His solution became known as Descartes' circle theorem. Philip Beecroft, an English amateur mathematician, rediscovered Descartes' circle theorem in 1842. Then it was discovered again in 1936 by Frederick Soddy (1877-1956), who had won a Nobel Prize in 1921 for his discovery of isotopes [8]. Soddy expressed the theorem in the form of a poem, "The Kiss Precise," which was published in the journal Nature and is included below. It may have been the flavor of the added poem that set Soddy apart from his predecessors, as the two circles are known today as the inner and outer Soddy circles. Additionally, Soddy extended the theorem to the analogous formula for six 
spheres in three dimensions [9, Soddy Circles].

Though Soddy provided an analytic solution to the kissing coins problem, we do not know whether he constructed a synthetic solution using compass and straightedge. For our part we find compass and straightedge solutions easier to understand than their analytic counterparts. This section is devoted to the Euler-GergonneSoddy triangle (EGST). Though it does not provide a solution to the kissing coins problem, it does provide a unique insight to finding the Soddy circles. Many of the connections between the EGST and the Soddy circles solution to the kissing coins problem were inspired by Oldknow's article [8], which uses trilinear coordinates, harmonic ranges, and parameterizations of lines to construct the EGST. It is interesting to note that Oldknow attributes many of his investigations to the use of geometric software packages, a current trend among geometry researchers.

As noted already, the kissing coins problem is the special case of Apollonius' problem where all three circles are mutually tangent. In this arrangement there are two solution circles, the inner and outer Soddy circles as shown in Figure 2. There are two questions of interest that arise from this special case: what are the radii of the Soddy circles and where are their centers located?

As a partial answer to the latter question, the Soddy centers lie on the line

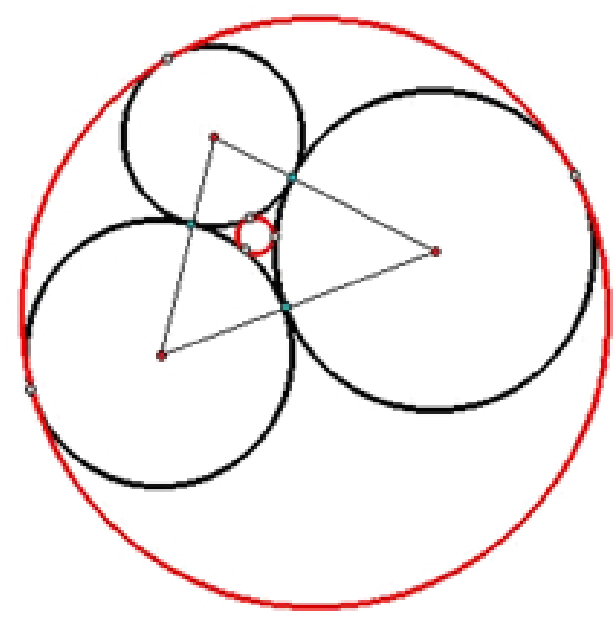

Figure 2. Soddy circles.

\section{The Kiss Precise}

For pairs of lips to kiss maybe Involves no trigonometry.

'Tis not so when four circles kiss

Each one the other three.

To bring this off the four must be

As three in one or one in three.

If one in three, beyond a doubt

Each gets three kisses from without.

If three in one, then is that one

Thrice kissed internally.

Four circles to the kissing come.

The smaller are the benter.

The bend is just the inverse of

The distance from the center.

Though their intrigue left Euclid dumb,

There's now no need for rule of thumb.

Since zero bend's a dead straight line

And concave bends have minus sign,

*The sum of the squares of all four bends

Is half the square of their sum.*

To spy out spherical affairs

An oscular surveyor

Might find the task laborious,

The sphere is much the gayer,

And now besides the pair of pairs

A fifth sphere in the kissing shares.

Yet, signs and zero as before,

For each to kiss the other four

*The square of the sum of all five bends

Is thrice the sum of their squares. *

Frederick Soddy (Nobel Prize, Chemistry, 1921) Nature, June 20, 1936 [10].

determined by the incenter and Gergonne point of the reference triangle. This line is known as the Soddy line and it contains one of the sides of the EGST. Given three vertices of a triangle, we show how to construct its EGST. The reader may want to refer to the Appendix for a glossary of geometric terms involved in this construction.

We start with the Euler line since it is the easiest to construct. Given a reference triangle, find the circumcenter as the intersection of the perpendicular bisectors and the orthocenter as the intersection of the altitudes. These two points determine the Euler line, which also 


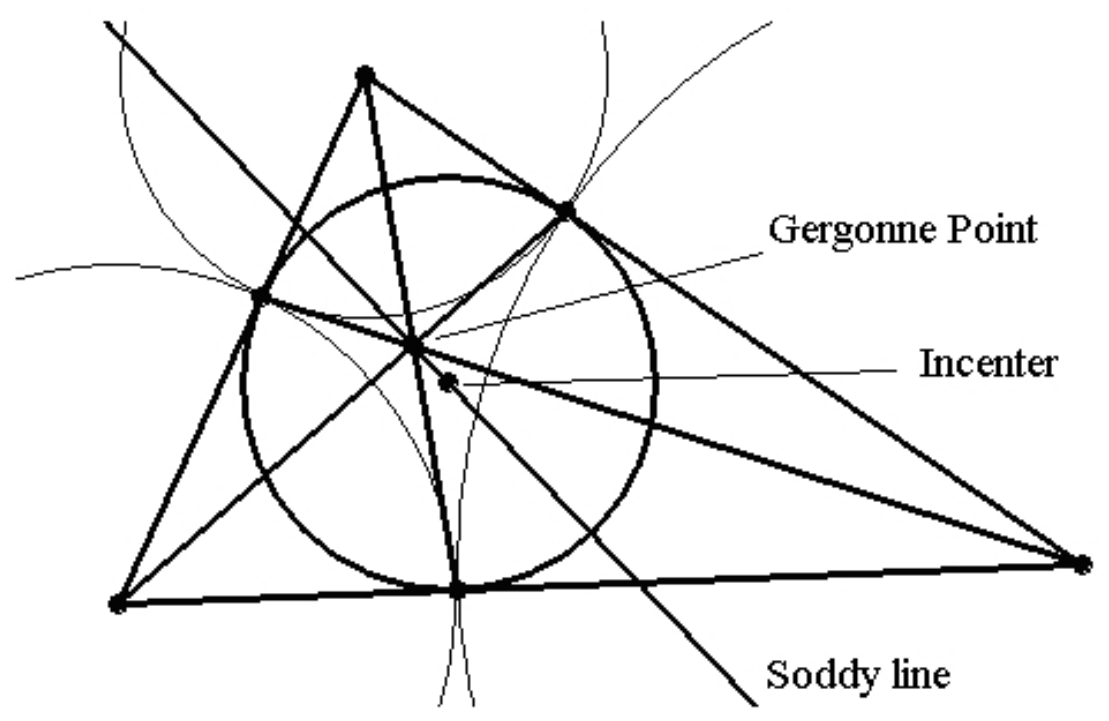

Figure 3. The Gergonne point, incenter, incircle and Soddy line.

contains the triangle's centroid (the intersection of the medians). The Euler line also contains a number of other important triangle centers including the center of the nine-point circle.

The Soddy line is determined by the incenter, where the angle bisectors coincide, and the Gergonne point. The incenter is the center of the unique circle that is internally tangent to its reference triangle at three points. These three points are called the contact points, and together they form the contact triangle of the reference triangle. The Gergonne point can most easily be found as follows: erect perpendiculars containing the incenter from each side of the reference triangle. These perpendiculars meet the reference triangle at the contact points. The lines containing the contact points and the opposite vertices on the reference triangle coincide at the Gergonne point (see Figure 3).

Importantly, the contact points have special meaning to the kissing coins problem. Given three noncollinear points, one can uniquely construct the three mutually tangent circles centered at these points. Construct the reference triangle with these vertices and its contact triangle, as described above; the mutually tangent circles are centered at the vertices of the reference triangle and contain the nearby vertices of the contact triangle! Hence, three non-collinear points uniquely determine the radii of the kissing coins.

To construct the Gergonne line, one must understand the notion of triangles in perspective. The idea of perspective was introduced in the Renaissance to create the idea of depth in art. The principle of perspective is that all lines meet at a point, thus providing depth. A well-known example of this is Leonardo da Vinci's Last Supper, where all the lines forming the walls, ceiling and edges of the table meet at a point of perspective, which happens to be the head of Christ.

The idea of perspective objects can be applied in geometry in relation to triangles. The two triangles $\triangle A B C$ and $\triangle A^{\prime} B^{\prime} C^{\prime}$ in Figure 4 are perspective from a line since the extensions of their three pairs of corresponding sides meet in collinear points $X, Y$, and $Z$. The line joining these points is called the perspectrix. It can also be said that two triangles are perspective from a point if their three pairs of lines joining their corresponding sides meet at a point of concurrence $O$. This point is called the perspector, perspective center, homology center, or pole [9, Perspective Triangles].

In the kissing coins problem the triangle formed by the centers of the circles and its contact triangle are perspective triangles. The perspector and the 


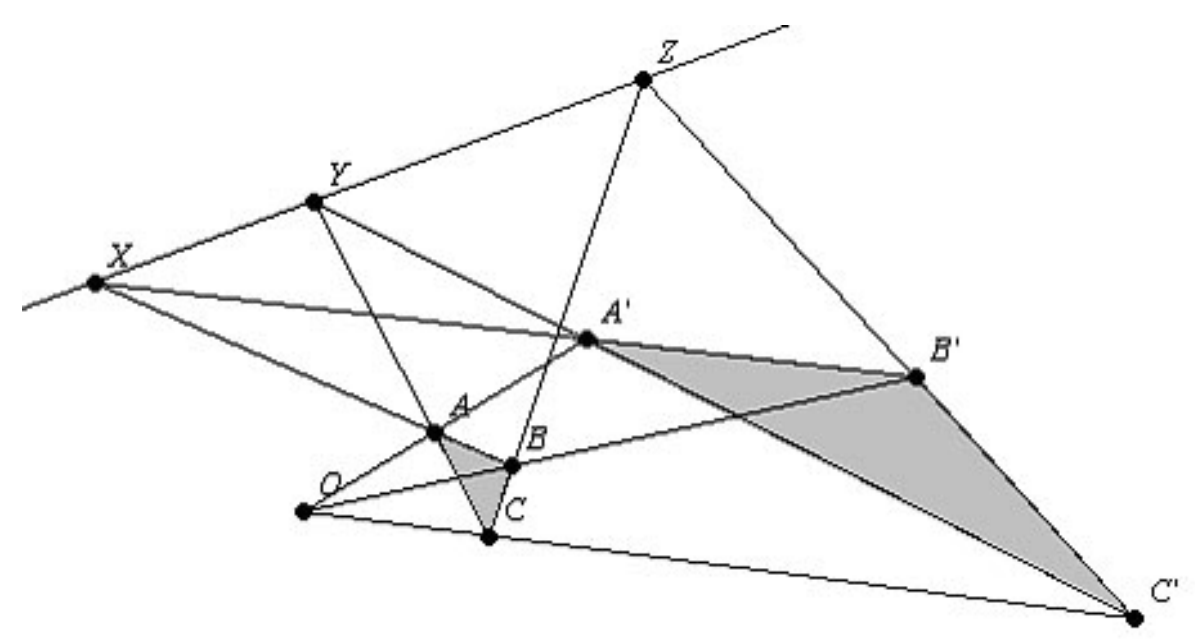

Figure 4. Triangles in perspective: Triangles $\triangle A B C$ and $\triangle A^{\prime} B^{\prime} C^{\prime}$ are perspective from a line since the extensions of their three pairs of corresponding sides meet in collinear points $X, Y$, and $Z$.

perspectrix of these two triangles are called the Gergonne point and Gergonne line, respectively, as shown in Figure 5.

The union of the Euler line, Soddy line and Gergonne line form the EGST, as seen in Figure 6. The vertices of EGST are known as the de Longchamps point, Evans point and Fletcher point. The Soddy line and Gergonne line always form a right angle at the Fletcher point [8, p. 328]. As mentioned previously the Soddy points, being the centers of the inner and outer Soddy circles, lie on the Soddy line and form a harmonic range with the incenter and Gergonne point [8, p. 326]. Further, the radii of both Soddy circles can be expressed in terms of ratios of the radii of the three given circles and the incircle. We will see in the next section that two of the solution circles to Apollonius' problem are centered on the Soddy line of the EGST.

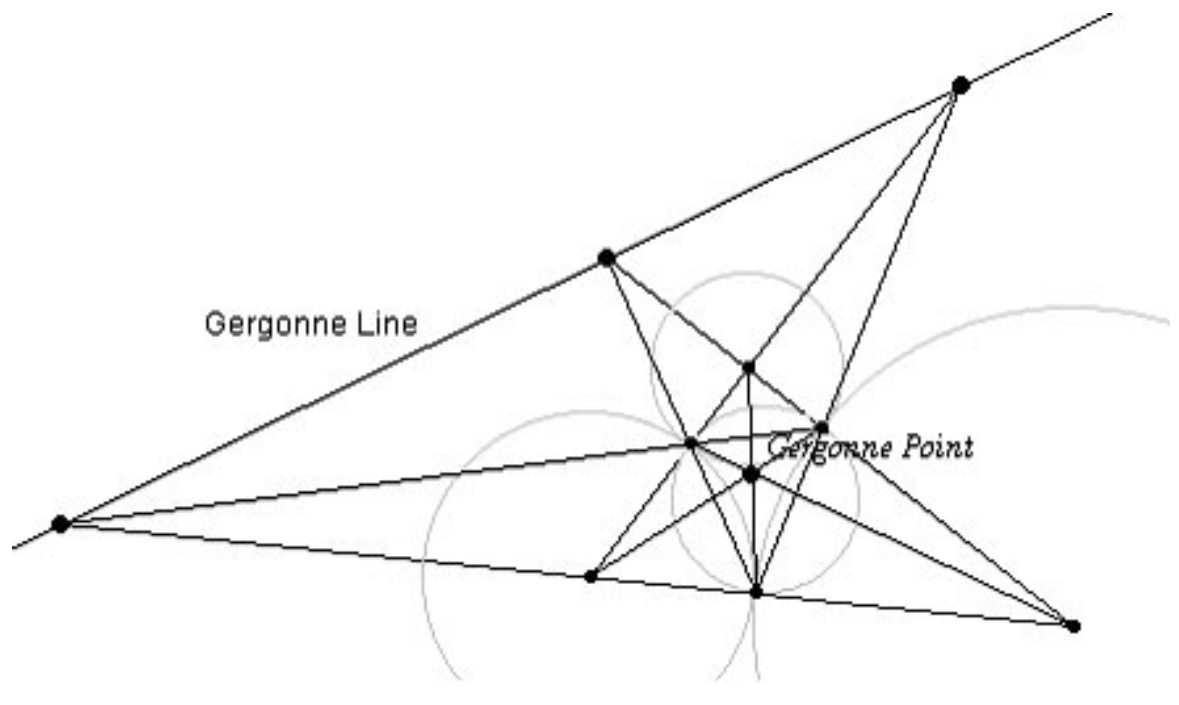

Figure 5. Gergonne point and line. 


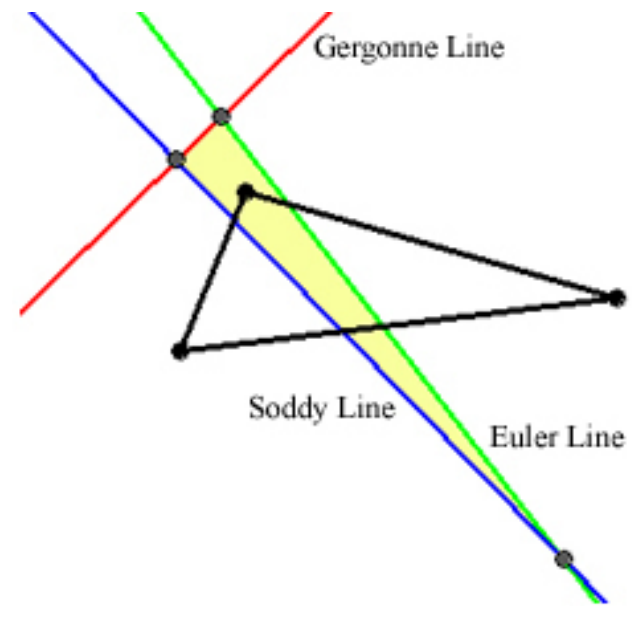

Figure 6. Euler-Gergonne-Soddy triangle.

\section{THE EPPSTEIN SOLUTION}

David Eppstein, a professor in the Department of Information and Computer Science at the University of California at Irvine, is a researcher in the areas of computational geometry and graph algorithms. Eppstein is also the founder and author of the popular Geometry Junkyard website. On this site Eppstein details a solution, discovered by Eppstein himself, that constructs Frederick Soddy's circles with a compass and straightedge. The basis of Eppstein's solution comes from an article in The American Mathematical Monthly [13]. In this article Eppstein proves that three lines through opposite points of tangency of any four mutually tangent spheres in threespace are coincident. A resulting corollary from this lemma is that three lines through opposite points of tangency of any four mutually tangent circles in the plane are coincident.

Eppstein's solution is as follows (see Figure 7a): form a triangle connecting the three circle centers and drop a perpendicular line from each center to the opposite triangle edge. Each of these lines cuts its circle at two points. Seen in Figure $7 \mathrm{~b}$, construct a line from each cut point to the point of tangency of the other two circles. These lines cut their circles in two more points, yielding six total, which are the points of tangency of the Soddy circles. Once these six points are known, the Soddy circles' centers are easily found to lie on the line determined by the incenter and Gergonne point, a.k.a. the Soddy line.

In Eppstein's solution, one may notice that there are two sets of three lines, as seen in Figure $7 b$, each intersecting at a common point. This is the result of the corollary from Eppstein's article mentioned above. Eppstein points out that despite their simplicity of definition and the large amount of study into triangle geometry, these two points do not appear in the list of over 1,000 known triangle centers collected by Clark Kimberling and Peter Yff [13, p. 65]. Thus, these two points have become known as the Eppstein points and, remarkably, they lie on the Soddy line and form a harmonic range with the Gergonne point and incenter [8, p. 327].

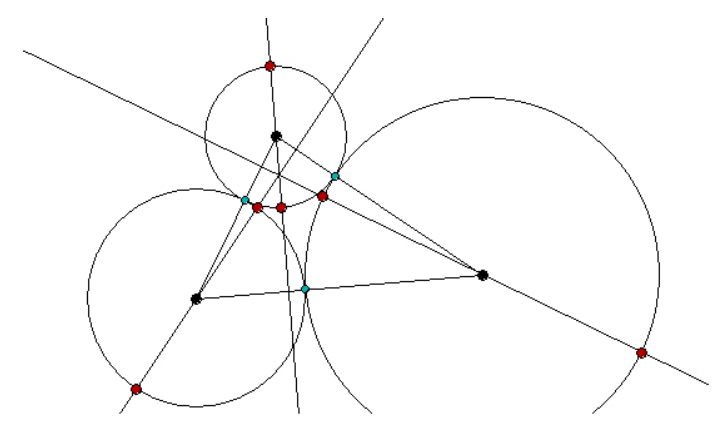

Figure 7a. The Eppstein solution.

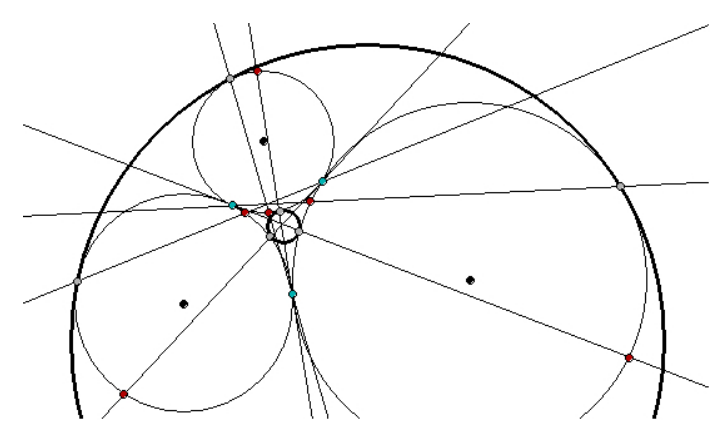

Figure 7b. Soddy circle and Eppstein points. 


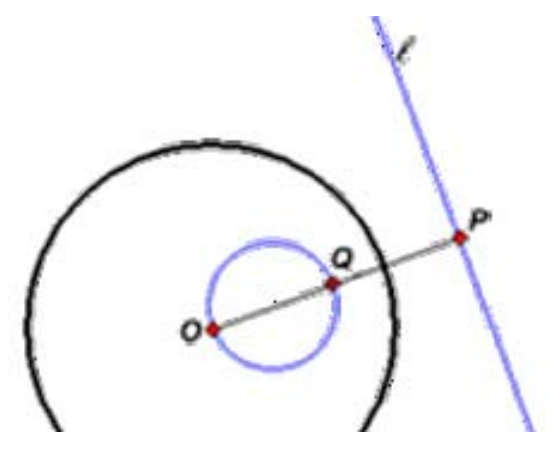

Figure 8. Inversion of line I through circle C.

\section{THE GERGONNE SOLUTION}

Joseph Diaz Gergonne was born in France in 1771 and died there in 1859. He spent most of his youth serving in the military until 1795. Afterwards, he began his mathematical study, which spawned a number of mathematical ideas but mostly focused on the area of geometry. Gergonne is well known for creating the journal officially called the Annales de Mathématique Pures et Appliquées but became known as Annales de Gergonne [14, p. 226]. His journal featured such prominent mathematicians as Jakob Steiner and Evariste Galois. Mentioned earlier are a few of the results of his work in the solution of the Soddy circles. Not only did Gergonne supply all eight solution circles to Apollonius' problem, he also introduced the word polar as it applies to inversion geometry.

Inversion geometry deals with transformations of the plane that leave a given circle fixed while taking its interior points to its exterior and vice versa. Not just the subject of advanced Euclidean geometry, inversion geometry arises in hyperbolic geometry and conformal mappings of the complex plane. While the details and intricacies of inversion geometry are beyond the scope of this paper, we do make use of the fact that we can invert any point through a given circle using compass and straightedge constructions [15].

For our purposes, we need the inversion geometry fact that a circle inversion through the circle $C$ of radius $r$ centered $\mathrm{O}$ in Figure 8 takes the line I to the circle with diameter $\overline{O Q}$. In this arrangement $P$ and $Q$ are on the line perpendicular to I containing $\mathrm{O}$, and
$(O P)(O Q)=r^{2}$. Points $P$ and $Q$ are easily constructed with compass and straightedge, and point $Q$ is called the inversion pole of the line $\mathrm{I}$.

Gergonne's solution requires constructing the dilation points for each pair of circles [16]. The dilation points of a pair of circles are the two points of central similarity about which one circle can be dilated (or contracted) to the other. As there are three pairs of circles with two dilation points each, this process yields six points. These lie three by three on four lines, forming a four-line geometry, as illustrated in Figure 9.

Determine the inversion poles of one of the dilation lines with respect to each of the three circles, as in Figure 10a, and connect the inversion poles with the radical center, as shown in Figure 10b. The radical center is the intersection of the three radical axes; the radical axis of two circles is the line that contains the center points of all

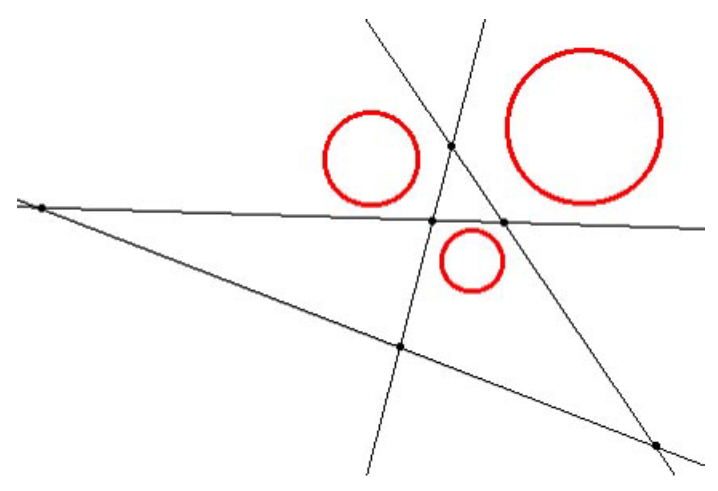

Figure 9. Dilation points and lines

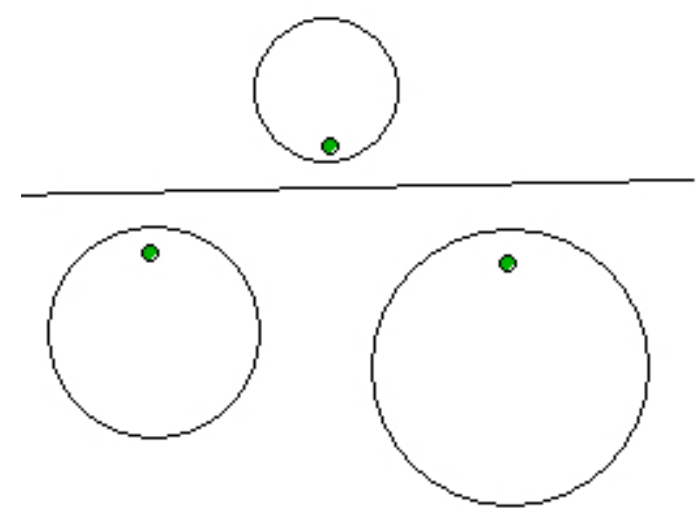

Figure 10a. Inversion poles 


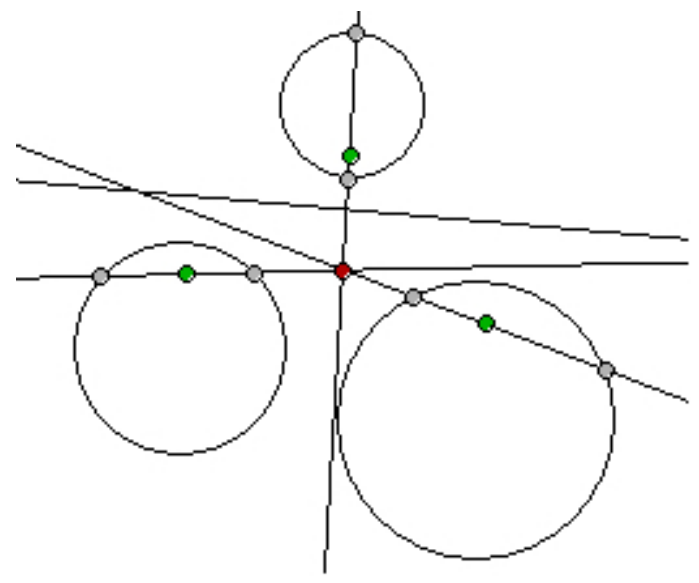

Figure 10b. Tangent points.

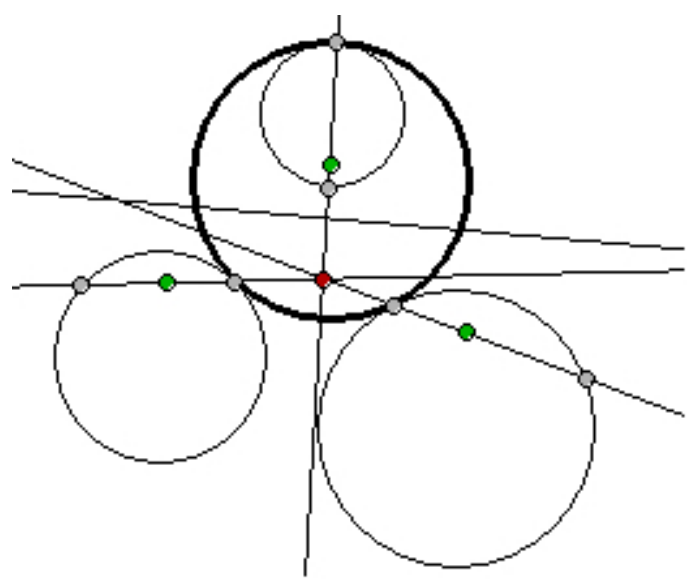

Figure 11a. Tangent circle.

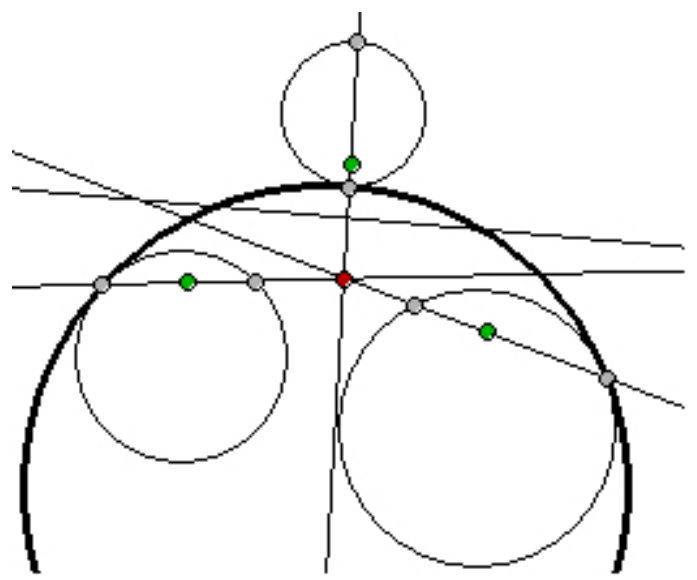

Figure 11b. Another tangent circle. that are orthogonal to both of the given circles. In this case, each line containing the radical center and an inversion pole intersects its respective circle at two points.

The center of the upper circle in

Figure $10 \mathrm{~b}$ is alone on one side of the dilation line formed by the dilation points. On that circle pick the intersection point furthest from the radical center. On the other two circles pick the near intersection points. These three points are the tangent points for the solution circle shown in Figure 11a. The other three intersection points are the tangent points for another solution circle, shown in Figure 11b.

This construction sequence yields all four lines formed from the dilation points, and for each line produces at most two solution circles. Thus, all eight solution circles can be constructed. We should mention that this process, while not terribly complicated, does require careful record keeping. When constructing Gergonne's solution, we used the interactive geometry software program Cinderella ${ }^{\mathrm{TM}}$. Cinderella ${ }^{\mathrm{TM}}$ allows one to hide lines and circles. Without this capability we do not believe that we could have constructed the solution. As pictured below in Figure 12, the solution becomes very cluttered when every line and circle is visible. We are truly impressed that Gergonne, or anyone before the computer age, could have the patience to perform this feat.

\section{CONNECTIONS AND EXTENSIONS}

In this section we observe some connections relating the EGST, Eppstein's solution and Gergonne's solution and suggest some routes for further study.

One of the most intriguing properties that we have found is the relation of the Gergonne line and the Gergonne solution. Since both share the name of J. D. Gergonne it might come as no surprise that they are related, but we do not consider this fact to be obvious. In Gergonne's solution one may recall that a line formed by the three outer dilation points, which we call the outer dilation line, is used to find two solution circles. In the special case where all three circles are mutually tangent, the Gergonne line and the outer dilation line coincide. Also, when the given circles are 


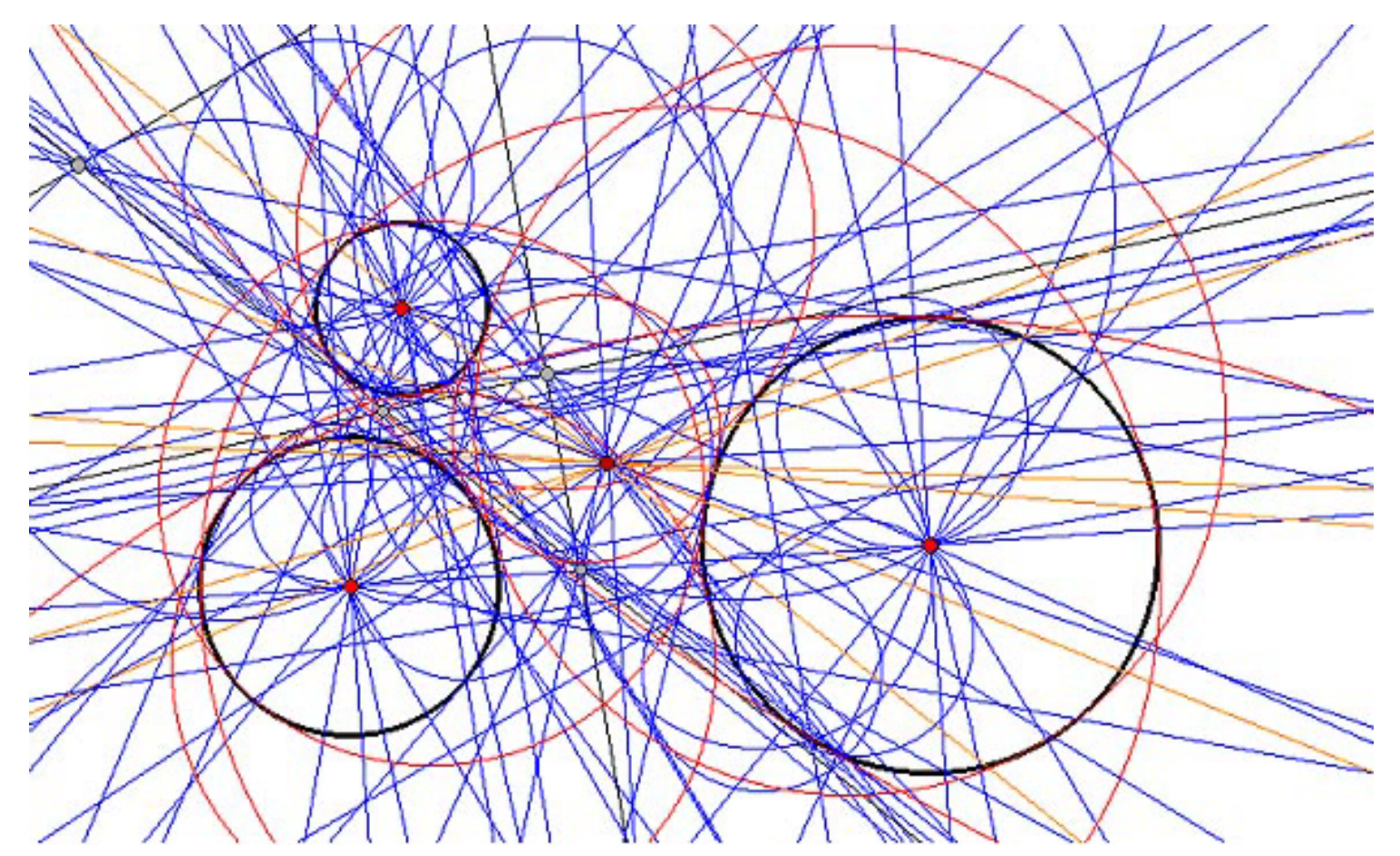

Figure 12. The Complete Gergonne Solution.

mutually tangent, the two solution circles formed by the outer dilation line are the Soddy circles. Thus, one begins to see the importance of the Euler-Gergonne-Soddy triangle and the role it plays in Apollonius' problem. Furthermore, if the three given circles are mutually tangent, the incenter of the reference triangle coincides with the radical center, and the radical axes that define the radical center are the perpendicular lines from the triangle sides to the incenter.

Until now we have allowed the reader to believe that Eppstein's solution to the kissing coins problem only solves for two of the solution circles whereas Gergonne's solution finds all eight solution circles to the general Apollonius' problem. The observant reader should ask: "Where are the remaining six tangent circles in the kissing coins arrangement?" It turns out that each of the three given circles represents two solutions. This can best be observed using geometry software to execute Gergonne's solution and arranging the three given circles to be nearly tangent. One will observe that the coins, now separated by a small distance from each other, are each internally tangent to two solution circles just slightly larger than the coin itself.

As another special case of three circles in general position, consider what happens when the radius of one of the given circles tends toward zero. Here, the eight solution circles collapse to four, and they do so in pairs. When the radius of a second circle decreases, the four solution circles collapse to two. Finally, when the radius of the third circle shrinks, the two solutions collapse to one. Figure 13 illustrates this nicely for a sequence of circles whose radii tend toward zero. Hence, Euclid's Proposition 5, Book IV, for finding the circumcenter of a triangle should really be viewed as a very special case of Gergonne's solution to Apollonius' problem!

As yet another special case, consider when the given circles are the excircles of a triangle. Here, the vertices of the triangle are the inner dilation points for the pairs of circles. Surprisingly, the lines extending the triangle sides are three of the Apollonius solutions; they are limiting cases as the radii of three of the solution circles tend to infinity! Feuerbach's Theorem guarantees that the nine-point circle is 

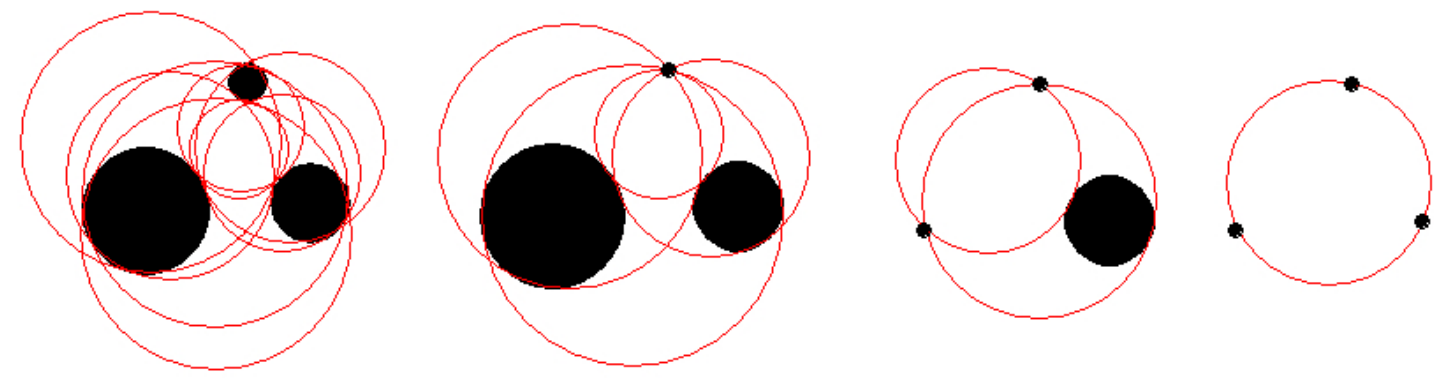

Figure 13. Shrinking circles and collapsing solutions.

simultaneously tangent to the three excircles, providing a fourth solution circle [17,p. 46 ]. This solution is analogous to the inner Soddy circle solution to the kissing coins problem in that the three excircles are externally tangent to the nine-point circle. A fifth solution circle lies so that the excircles are internally tangent, and the remaining three solution circles each have one excircle internally tangent and the other two excircles externally tangent.

With regard to further study, the interested reader may wish to pursue the following topics.

In Apollonius' problem the three given objects are taken from among circles, points and lines. The latter two objects should be thought of as limiting cases when the radius of a circle approaches zero or infinity. Gergonne's solution only applies to the case when all three objects are circles since circle inversion is used. Rather than use three circles, one may wish to examine all combinations of points, lines and circles, decide for which configurations all eight solution circles appear, and use geometry software to construct solution circles. For example, no solution circles exist in the case of three parallel lines, and two solution circles exist for two parallel lines cut by a transversal. Are there any connections to Gergonne's solution?

It is also worth exploring the special case of three circles centered at points that are the vertices of an isosceles or an equilateral triangle. If at least two of the three sides of the reference triangle are congruent, the Euler line and the Soddy line coincide, so the EGST is degenerate. In the context of Eppstein's solution, this corresponds to kissing coins with equal radii.
Given three noncollinear points, consider all sets of three non-overlapping circles centered at these points. What are the possible locations for the centers of the tangency circles for which the three circles are either all internally tangent or all externally tangent? The kissing coins problem is the special case when the three circles centered at the triangle vertices are tangent to each other. In this instance, perhaps the inner and outer Soddy centers that solve this problem are known triangle centers.

Finally, as noted in section 2, the EGST is always a right triangle. A benefit of Cinderella ${ }^{\mathrm{TM}}$ is the ability to dynamically move elements while preserving the incident relations among points, lines and circles. With this capability the vertices of the original triangle can be moved freely, and we have observed that the EGST always appears to be long and skinny (see Figure 6). The maximum value of the angle between the Euler line and Gergonne line is 90 degrees, but what is the smallest possible value of this angle?

\section{REFERENCES}

1. Boyer, C. B. A History of Mathematics. New York: John Wiley \& Sons, 1968.

2. Boyer, C. B.; revised by Merzbach, Uta. A History of Mathematics. New York: John Wiley \& Sons, 1989.

3. Cajori, Florian. A History of Mathematics. New York: Chelsea Publishing Company, 1980.

4. Gow, James. A Short History of Greek Mathematics. New York: G. E. Stechert \& Co., 1923. 
5. Joyce, David E., "Euclid's Elements." http://aleph0.clarku.edu/ djoyce/java/ele ments/elements.html.

6. Heath, Thomas. A History of Greek Mathematics. New York: Oxford University Press, Vol. 2, 1921.

7. Court, N. A. "Historically Speaking, The Problem of Apollonius." The Mathematics Teacher. October 1961, pp. 444-452.

8. Oldknow, A. "The Euler-GergonneSoddy Triangle of a Triangle." The American Mathematical Monthly 103 (1996), pp. 319-329.

9. Weisstein, Eric W. Eric Weisstein's World of Mathematics. http://mathworld.wolfram.com.

10. Soddy, F. "The Kiss Precise." Nature 137 (1936), p. 1021.

11. Eppstein, David. "Tangencies: Apollonian Circles." Geometry Junkyard. www.ics.uci.edu/ eppstein/junkyard/tan gencies/apollonian.html.
12. Bogomolny, Alexander. "Apollonius Problem: What is it?" Cut The Knot! www.cut-the-

knot.com/Curriculum/Geometry/Apolloni us.shtml.

13. Eppstein, David. "Tangent Spheres and Triangle Centers." The American Mathematical Monthly 108 (2001), pp. 63-66.

14. Boyer, C. B. History of Analytic Geometry. New York: Scripta Mathematica, 1956.

15. Goodman-Strauss, Chaim. "Compass and Straightedge in the Poincare Disk." The American Mathematical Monthly 108 (2001), pp. 38-49.

16. Kunkel, Paul. "Tangent Circles." http://whistleralley.com/tangents/tangent s.htm.

17. Baragar, Arthur. A Survey of Classical and Modern Geometries with Computer Activities. New Jersey: Prentice-Hall Inc. 2001.

APPENDIX: Glossary of Terms

\begin{tabular}{|l|l|}
\hline \multicolumn{2}{|c|}{ Points and Centers } \\
\hline Centroid & The intersection of the medians \\
\hline Circumcenter & The intersection of the perpendicular bisectors \\
\hline Incenter & Intersection of the angle bisectors \\
\hline Orthocenter & Intersection of the altitudes \\
\hline Gergonne point & Perspector of a reference triangle and its contact triangle \\
\hline Evans point & Intersection of the Euler and Soddy lines \\
\hline Fletcher point & Intersection of the Soddy and Gergonne lines \\
\hline De Longchamps & Intersection of the Euler and Gergonne lines \\
\hline \multicolumn{2}{|l|}{ Lines } \\
\hline Euler line & $\begin{array}{l}\text { Defined by the circumcenter and orthocenter; also contains the } \\
\text { centroid and nine-point center }\end{array}$ \\
\hline Gergonne line & Line of perspective for a reference triangle and its contact triangle \\
\hline Soddy line & $\begin{array}{l}\text { Defined by the incenter and Gergonne point; also contains the } \\
\text { Eppstein and Soddy points }\end{array}$ \\
\hline \multicolumn{2}{|l|}{ Circles } \\
\hline Circumcircle & Unique circle containing a triangle's vertices \\
\hline Incircle & Unique circle internally tangent to all three triangle sides \\
\hline Nine-point circle & $\begin{array}{l}\text { Contains side midpoints, feet of altitudes, and midpoints of } \\
\text { segments joining the orthocenter to the vertices }\end{array}$ \\
\hline Inner Soddy circle & Circle that is internally tangent to the kissing coins \\
\hline Outer Soddy circle & Circle that is externally tangent to the kissing coins \\
\hline \multicolumn{2}{|l|}{ Triangles } \\
\hline Contact triangle & $\begin{array}{l}\text { Defined by the points of tangency of reference triangle and its } \\
\text { incircle; vertices are where the kissing coins touch }\end{array}$ \\
\hline $\begin{array}{l}\text { Euler-Gergonne-Soddy } \\
\text { triangle }\end{array}$ & \begin{tabular}{l} 
Triangle formed by the Euler, Gergonne and Soddy lines \\
\hline
\end{tabular} \\
\hline
\end{tabular}


